\title{
3D User Interfaces for Interactive Annotation of Vascular Structures
}

\author{
Patrick Saalfeld, Sylvia Glaßer, Bernhard Preim \\ Visualization Group, Faculty of Computer Science, Otto-von-Guericke University of Mag- \\ deburg, Germany
}

\begin{abstract}
A large number of scientific documents show additional information through annotations, which can also be used in interactive 3D environments. Here, labels are usually placed on an image plane and do not benefit from the 3D domain to convey, e.g., depth cues. These are especially important for spatially complex structures, such as the Circle of Willis, which is the central part of the cerebral vessel system. We present an approach for diegetic annotations, i.e., labels which are part of the 3D world. Based on the wide space for possible label positions and orientations, we enable the user to interactively create and position labels. For this, we present a concept for a 3D User Interface (3D UI) setup in a semiimmersive and a fully-immersive environment. Furthermore, we describe an evaluation design to evaluate the different setups.
\end{abstract}

\section{Introduction}

Annotations provide additional information in a large number of scientific documents. Besides static visualizations, labels in interactive environments can also be used to increase the efficiency of learning, e.g., in the medical domain (Hartmann et al. 2005). Here, physicians and medical students can be supported by exploring and inspecting labeled structures in medical data sets. Several algorithms have been proposed to position labels automatically. Even for interactive 3D applications, the positioning is realized in the 2D domain, which takes the current rendered view and applies image-based approaches.

We represent annotations as a diegetic interface (Schild et al. 2013). There, labels are part of the 3D space to support immersion. Since the problem to find the optimal position and orientation for diegetic annotations is NP-hard, this work focuses on user interaction to create, place and delete annotations (Harmon et al. 1996). To support the immersion and depth perception, a 3D UI is used. We present a concept to compare two output modalities: a stereoscopic display and a virtual reality (VR) head-mounted display (HMD). For both modalities, the same interaction techniques realized with stylus-based ray casting are used. 
As a test scenario, we annotate the Circle of Willis, a vessel structure in the brain which is usually illustrated as a 2D unfolded and flat representation. The Circle of Willis is ideal for diegetic annotations in an immersive environment for two reasons: first, it contains many different sub-structures and secondly, due to spatially complex vascular extents and transitions, it is difficult to comprehend its non-flat real anatomical representation.

\section{Related Work}

Besides an internal labeling, annotations can be placed external beneath structures connected to the label with a line. The annotations can be arranged with different layouts, e.g., circular layouts (Hartmann et al. 2005). In augmented reality (AR) or VR environments the integration of 2D meta-data, such as images and texts, is described with the term view management (Tatzgern et al. 2014). In contrast to 2D approaches where annotations are usually placed on the image plane, the view management aims at integrating the labels in the $3 \mathrm{D}$ environment to maintain immersion. An approach for automatic label placement for diegetic annotations with an immersive multiscreen display system is described by Pick et al. (2010). Similar, the focus of our work is the placement of labels in the 3D environment with an exception: we integrate the user in the placement process instead of using a layout algorithm.

\section{Materials and Methods}

In this section, we will provide short background information about the Circle of Willis, explain our user interface (UI) setup and the evaluation concept.

The Circle of Willis. The treatment of cerebrovascular diseases (including stroke and atherosclerosis) is a substantial part of clinical practice and requires excellent knowledge of patient-individual morphology by the clinical experts. The central part of the cerebral vessel system is the Circle of Willis (Fig. 1). The Circle of Willis comprises a combination of arteries supplying the brain with variations w.r.t. to shape and length. Thus, it is an excellent example for the challenging patient-individual 3D anatomy (Fig. 1(c)). Medical students have to rely on conventional 2D illustrations or anatomical preparations which are not sufficient to represent the spatially complex vascular extents and transitions.

3D User Interface Setup \& Interaction Techniques. 3D UIs can be classified according to three components: output and input devices and interaction techniques. For the output, we compare two devices. First, the semi-immersive zSpace (zSpace Inc., San Francisco, USA) which has a $120 \mathrm{~Hz}$ stereoscopic display and emits polarized images for passive glasses. The glasses are tracked in six degrees of freedom (DOF) through infrared (IR) markers and allow head tracking. The second device is the fully-immersive Oculus Rift DK2 (Oculus, California, USA) VR HMD with a $75 \mathrm{~Hz}$ display. Positional tracking is realized with build-in sen sors as well as an external camera. As input, a stylus with buttons from the zSpace is used for 

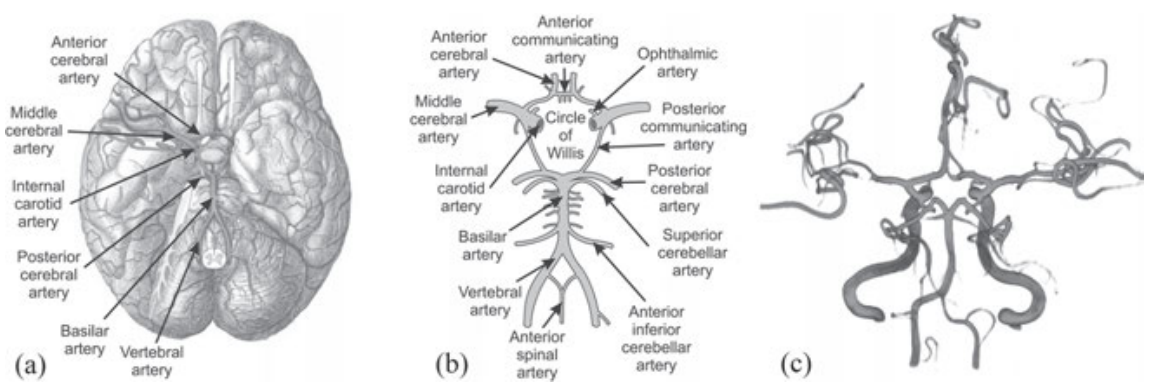

Figure 1: The blood supply of the brain is maintained by the anterior and the posterior cerebral circulation, see (a), and the Circle of Willis, see (b), as a backup circulation (adapted from illustrations of Gray (1918)). The 3D volume rendering in (c) reveals the complex spatial shape of the Circle of Willis.

both output modalities. The stylus is tracked from the zSpace with IR LEDs and allows six DOF interaction. For the zSpace output, the orientation of the stylus is virtually extended in the virtual scene represented with a visible ray. For the Oculus Rift, a 3D representation of the stylus is used in VR which is also extended with a virtual ray.

We want to realize basic interaction techniques, i.e., creation, placement, swapping and deletion of annotations. For creating labels, the stylus is pointed to a structure, such that the virtual ray intersects it (Fig. 2 (a)). Now, a button is pressed which determines the anchor position of the label. If the user releases the button, the label is created beneath the structure. Alternatively, the user can hold and drag to place the annotation to a preferred position. The label is first created with a name stored in the structure, then connected to the anchor point with a line and after that oriented view-aligned to the user (Fig. 2 (b)). To reposition a label the label can be dragged to a new position. To swap labels, which is useful if two connection lines intersect, one label is dragged and released over another label. Deleting an annotation can be realized by using the virtual ray as a knife and cutting connection lines.
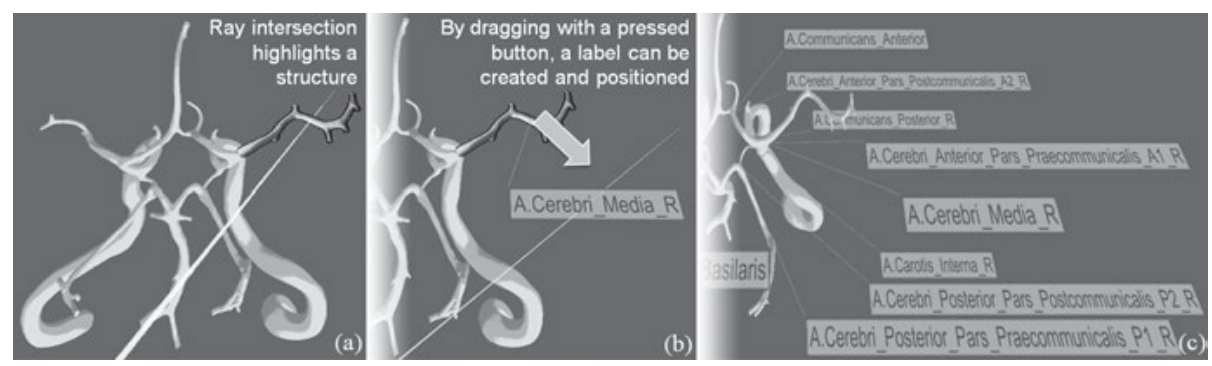

Figure 2: By intersecting a structure with the stylus ray and pressing a button, a label of this structure is created (a) which can be dragged to the desired position $(b)$. (c) shows the Circle of Willis with arranged annotations.

Evaluation Design. Due to the two output modalities, the intensity of immersion and the interaction experience differ. For the stereoscopic display, the user sees the physical stylus but interacts with the virtually extended ray. The HMD, however, shows the stylus as a virtual representation. Therefore, we want to analyze if there are differences regarding task 
performance and user performance metrics. We let the user test both output devices in a crossover design. For tasks performing metrics, the time to perform interaction techniques is taken as well as accuracy measures. The participant places a label as accurate as possible on a predefined position. Here, differences due to depth perception could arise. For user performance metrics, questionnaires are used to compare the usability of both devices as well as special 3D UI aspects such as presence and user comfort (Bowman et al. 2004).

\section{Discussion}

The presented concept for diegetic annotations is able to help medical students as well as physicians. Students are supported in the process of learning different structures of the Circle of Willis on a correct anatomical 3D model instead of an unfolded 2D version. Although the unfolded representation has advantages w.r.t. clarity, it is not sufficient to represent the spatially complex vascular structures. By providing a 3D UI, the understanding of vessel transitions and extent of the Circle of Willis is more adequate. The described evaluation allows the comparison of different output devices and their influence on interaction.

\section{Acknowledgement}

This work was partly funded by the German Federal Ministry of Education and Research (BMBF) within the Forschungscampus STIMULATE under grant number '13GW0095A'.

\section{References}

Bowman, D.A., Kruijff, E., LaViola, J.J., \& Poupyrev, I. (2004). 3D User Interfaces: Theory and Practice. Addison Wesley Longman Publishing Co., Inc., USA.

Gray, H. (1918). Anatomy of the Human Body. Philadelphia: Lea \& Febiger.

Hartmann, K., Götzelmann, T., Ali, K. \& Strothotte, T. (2005). Metrics for Functional and Aesthetic Label Layouts. Smart Graphics. Heidelberg: Springer, pp. 115-126.

Harmon, R., Patterson, W., Ribarsky, W. \& Bolter, J., (1996). The Virtual Annotation System. Virtual Reality Annual International Symposium. pp. 239-245.

Pick, S., Hentschel, B., Tedjo-Palczynski, Wolter, M. \& Kuhlen, T. 2010. Automated Positioning of Annotations in Immersive Virtual Environments. Eurographics Conference on Virtual Environments. Aire-la-Ville: Eurographics Association. pp. 1-8.

Schild, J., Bölicke, L., LaViola, J.J. \& Masuch, M. (2013). Creating and Analyzing Stereoscopic 3D Graphical User Interfaces in Digital Games. Human Factors in Computing Systems (SIGCHI). Paris: ACM, pp. 169-178.

Tatzgern, M., Kalkofen, D., Grasset, R. \& Schmalstieg, D. (2014). Hedgehog Labeling: View Management Techniques for External Labels in 3D Space. Virtual Reality (VR), Minneapolis: IEEE, pp. 27-32.

\section{Contact information}

saalfeld@isg.cs.uni-magdeburg.de 